\title{
Anti-CD33/CD3 BiTE Antibody AMG 673
}

National Cancer Institute

\section{Source}

National Cancer Institute. Anti-CD33/CD3 BiTE Antibody AMG 673. NCI Thesaurus. Code C147026.

A bispecific T-cell engager (BiTE) antibody composed of two single-chain variable fragments (scFv), one directed against the tumor-associated antigen (TAA) CD33 fused to one that is directed against the CD3 antigen found on T-lymphocytes, with potential immunostimulating and antineoplastic activities. Upon administration, anti-CD33/CD3 BiTE antibody AMG 673 binds to both the CD3 antigen on cytotoxic T-lymphocytes (CT Ls) and the CD33 antigen found on CD33-expressing tumor cells. This activates and redirects CT Ls to CD33-expressing tumor cells, which results in the CT L-mediated cell death of CD33-expressing tumor cells. CD33, a myeloid differentiation antigen, is expressed on normal non-pluripotent hematopoietic stem cells and overexpressed on a variety of cancer cell types, including acute myeloid leukemia (AML). It plays a key role in tumor initiation, proliferation and progression. 